\title{
A PROBLEM IN DIOPHANTINE APPROXIMATION FOUND IN RAMANUJAN'S LOST NOTEBOOK
}

\author{
BRUCE C. BERNDT AND SUN KIM
}

\section{INTRODUCTION}

We examine an untitled partial manuscript published on pages 262-265 with the Narosa edition of Ramanujan's lost notebook [3]. At the top of page 262 are two appended notes. The first, possibly in the handwriting of G.H. Hardy's former research student, Gertrude Stanley, reads (in part) "Paper a little difficult to understand after the first page." The second, definitely in the handwriting of Hardy, surmises "Odd problem. I don't profess to know whether there's much to it." On these aforementioned pages, Ramanujan considers the problem of approximating a certain quadratic irrational by rational numbers $n / m$ for a fixed but arbitrary positive integer $m$, $1 \leq m \leq \infty$. We do not know what motivated Ramanujan to consider this particular problem, which we describe in the next two sections. Nonetheless, the analysis is appealing and intriguing phenomena arise, especially in regard to the periodicity of approximations in Section 3. Indented passages below are quoted from Ramanujan's partial manuscript.

\section{A Diophantine Problem}

Let us consider the maximum of

$$
\epsilon_{m}\left(1-\epsilon_{m}\right)\left(1-2 \epsilon_{m}\right)
$$

where $\epsilon_{m}$ is a positive proper fraction and $m$ and $m \epsilon_{m}$ are positive integers. Let $v_{m}$ be the maximum of (2.1). If we do not assume that $m \epsilon_{m}$ is rational, we get that

$$
\epsilon_{m}=\frac{3-\sqrt{3}}{6}, \quad v_{m}=\frac{1}{6 \sqrt{3}} .
$$

Here, as a positive proper fraction, Ramanujan intends $\epsilon_{m}$ to be a rational number (not necessarily in lowest terms) with denominator $m$. If

$$
f(x):=x(1-x)(1-2 x)=x-3 x^{2}+2 x^{3},
$$

then it is easily checked that its critical points are $(3 \pm \sqrt{3}) / 6$. Since $f^{\prime \prime}(x)=-6+12 x$, we easily find that $(3+\sqrt{3}) / 6$ yields a local minimum and $(3-\sqrt{3}) / 6$ yields a local maximum. Ramanujan desires to approximate $(3-\sqrt{3}) / 6$ by a rational number $\epsilon_{m}$ with denominator not exceeding $m$. He then wants to calculate the corresponding value $f\left(\epsilon_{m}\right)=: v_{m}$.

We again record what Ramanujan has written. 
Now let

$$
m \cdot\left(\frac{3-\sqrt{3}}{6}\right)-\epsilon
$$

be an integer where $\epsilon$ is a positive proper fraction. Then we see from (2.2) that $\epsilon_{m}$ is either

$$
\frac{m \cdot\left(\frac{3-\sqrt{3}}{6}\right)-\epsilon}{m} \text { or } \quad \frac{m \cdot\left(\frac{3-\sqrt{3}}{6}\right)+1-\epsilon}{m} .
$$

In contrast to Ramanujan's first use of the term, positive proper fraction, $\epsilon$ is not rational but necessarily irrational, if $\epsilon_{m}$ is to be rational as required. Thus, $\epsilon$ is a positive (necessarily irrational) number, $0<\epsilon<1$, chosen so that the two values in (2.3) give the best two rational approximations to $(3-\sqrt{3}) / 6$ with denominator $m$. In the first instance of (2.3), the approximation is from below, while in the second instance, the approximation is from above.

Ramanujan then claims that if

$$
\epsilon_{m}=\frac{m \cdot\left(\frac{3-\sqrt{3}}{6}\right)-\epsilon}{m}, \quad \text { then } \quad v_{m}=\frac{1}{6 \sqrt{3}}-\frac{\epsilon^{2}}{m^{2}} \sqrt{3}-2 \frac{\epsilon^{3}}{m^{3}},
$$

and if

$$
\epsilon_{m}=\frac{m \cdot\left(\frac{3-\sqrt{3}}{6}\right)+1-\epsilon}{m}, \quad \text { then } \quad v_{m}=\frac{1}{6 \sqrt{3}}-\frac{(1-\epsilon)^{2}}{m^{2}} \sqrt{3}+2 \frac{(1-\epsilon)^{3}}{m^{3}} .
$$

With the use of (2.1), both of these calculations are straightforward.

We note that by replacing $\epsilon$ by $\epsilon-1$ in the value of $v_{m}$ in (2.4), we obtain the value of $v_{m}$ in $(2.5)$

Ramanujan then determines which of the two values of $v_{m}$ given in (2.4) and (2.5) is the larger. An elementary calculation shows that

$$
\frac{1}{6 \sqrt{3}}-\frac{\epsilon^{2}}{m^{2}} \sqrt{3}-2 \frac{\epsilon^{3}}{m^{3}}>\frac{1}{6 \sqrt{3}}-\frac{(1-\epsilon)^{2}}{m^{2}} \sqrt{3}+2 \frac{(1-\epsilon)^{3}}{m^{3}}
$$

if and only if

$$
6 \epsilon^{2}+(2 m \sqrt{3}-6) \epsilon+2-m \sqrt{3}<0 .
$$

It is easily checked that the roots of $6 \epsilon^{2}+(2 m \sqrt{3}-6) \epsilon+2-m \sqrt{3}=0$ are

$$
r_{1}, r_{2}:=\frac{1}{2}+\frac{-m \pm \sqrt{m^{2}-1}}{2 \sqrt{3}}, \quad \text { with } r_{2}<r_{1} .
$$

Thus, (2.6) is true if and only if $r_{2}<\epsilon<r_{1}$. Since the root that we seek is $r_{1}$, in conclusion, we see that:

$$
\text { If } \quad \epsilon<\frac{1}{2}-\frac{m-\sqrt{m^{2}-1}}{2 \sqrt{3}}, \quad(2.4) \text { is greater; }
$$




$$
\text { if } \quad \epsilon>\frac{1}{2}-\frac{m-\sqrt{m^{2}-1}}{2 \sqrt{3}}, \quad(2.5) \text { is greater; }
$$

and

$$
\text { if } \quad \epsilon=\frac{1}{2}-\frac{m-\sqrt{m^{2}-1}}{2 \sqrt{3}}, \quad(2.4) \text { and (2.5) are the same. }
$$

Now, if

$$
0<\epsilon<\frac{1}{2}-\frac{m-\sqrt{m^{2}-1}}{2 \sqrt{3}},
$$

then

$$
\frac{m-1}{2}-\sqrt{\frac{m^{2}-1}{12}}<m \epsilon_{m}<m \frac{3-\sqrt{3}}{6}<\frac{m+1}{2}-\sqrt{\frac{m^{2}-1}{12}}
$$

Also, if

$$
\frac{1}{2}-\frac{m-\sqrt{m^{2}-1}}{2 \sqrt{3}}<\epsilon<1
$$

then

Thus, if

$$
\frac{m-1}{2}-\sqrt{\frac{m^{2}-1}{12}}<m \frac{3-\sqrt{3}}{6}<m \epsilon_{m}<\frac{m+1}{2}-\sqrt{\frac{m^{2}-1}{12}} .
$$

we conclude that

$$
\epsilon \neq \frac{1}{2}-\frac{m-\sqrt{m^{2}-1}}{2 \sqrt{3}}
$$

$$
\epsilon_{m}=\frac{1}{m}\left[\frac{m+1}{2}-\sqrt{\frac{m^{2}-1}{12}}\right] .
$$

We also note that for those values of $m$ for which

$$
\epsilon=\frac{1}{2}-\frac{m-\sqrt{m^{2}-1}}{2 \sqrt{3}},
$$

by (2.10), we can choose either one from (2.3) for $\epsilon_{m}$. Thus,

$$
\epsilon_{m}=\frac{1}{m}\left(\frac{m-1}{2}-\sqrt{\frac{m^{2}-1}{12}}\right) \quad \text { or } \quad \frac{1}{m}\left(\frac{m+1}{2}-\sqrt{\frac{m^{2}-1}{12}}\right) .
$$

We remark that, by (2.3) and (2.12), we do not need greatest integer functions in (2.13). Hence, the formula in (2.11) is valid for all values of $m$, and in particular, we have alternate choices for $\epsilon_{m}$ in the case of (2.12). Therefore, we use (2.11) to calculate $\epsilon_{m}$. We then return to $(2.1)$ to determine $v_{m}$.

Ramanujan concludes page 262 with a table of the first ten values of $\epsilon_{m}$ :

$$
\begin{aligned}
& v_{1}=0, \quad v_{2}=0, \quad v_{3}=\frac{2}{3^{3}}, \quad v_{4}=\frac{6}{4^{3}}, \quad v_{5}=\frac{12}{5^{3}}, \quad v_{6}=\frac{20}{6^{3}}, \\
& v_{7}=\frac{30}{7^{3}}, \quad v_{8}=\frac{48}{8^{3}}, \quad v_{9}=\frac{70}{9^{3}}, \quad v_{10}=\frac{96}{10^{3}}, \quad \ldots
\end{aligned}
$$

In Table 1 , we list the values of $\epsilon_{m}$ for each $m, 1 \leq m \leq 10$, which were obtained from (2.11) or (2.13). We also add the corresponding values of $\epsilon$ in the table. 


\begin{tabular}{|r|c|c|c|}
\hline$m$ & $\epsilon_{m}$ & value of $\epsilon$ & $v_{m}$ \\
\hline 1 & 0,1 & $\frac{1}{2}-\frac{1}{2 \sqrt{3}}$ & 0 \\
\hline 2 & $0, \frac{1}{2}$ & $\frac{3-\sqrt{3}}{3}$ & 0 \\
\hline 3 & $\frac{1}{3}$ & $\frac{3-\sqrt{3}}{2}$ & $\frac{2}{27}$ \\
\hline 4 & $\frac{1}{4}$ & $2-\frac{2 \sqrt{3}}{3}$ & $\frac{3}{32}$ \\
\hline 5 & $\frac{1}{5}$ & $\frac{3}{2}-\frac{5 \sqrt{3}}{6}$ & $\frac{12}{125}$ \\
\hline 6 & $\frac{1}{6}$ & $2-\sqrt{3}$ & $\frac{20}{6^{3}}$ \\
\hline 7 & $\frac{1}{7}, \frac{2}{7}$ & $\frac{5}{2}-\frac{7 \sqrt{3}}{6}$ & $\frac{30}{7^{3}}$ \\
\hline 8 & $\frac{1}{4}$ & $3-\frac{4 \sqrt{3}}{3}$ & $\frac{3}{32}$ \\
\hline 9 & $\frac{2}{9}$ & $\frac{7}{2}-\frac{3 \sqrt{3}}{2}$ & $\frac{70}{3^{6}}$ \\
\hline 10 & $\frac{1}{5}$ & $3-\frac{5 \sqrt{3}}{3}$ & $\frac{12}{5^{3}}$ \\
\hline
\end{tabular}

TABLE 1. Table of Values for $v_{m}, 1 \leq m \leq 10$

At the top of page 263, Ramanujan remarks that, by (2.4) and (2.5) (to which one might also add (2.8) and (2.9)),

the order of $v_{m}$ is minimum when

$$
\epsilon=\frac{1}{2}-\frac{m-\sqrt{m^{2}-1}}{2 \sqrt{3}}
$$

that is the minimum order of

$$
v_{m}=\frac{m^{2}-4}{6 m} \sqrt{\frac{m^{2}-1}{3}}
$$

and the corresponding value of

$$
\epsilon_{m}=\frac{1}{m}\left(\frac{m+1}{2}-\sqrt{\frac{m^{2}-1}{12}}\right) .
$$

Note that the corresponding minimum order of $v_{m}$ can be determined from either (2.4) or (2.5). Several remarks need to be made. Ramanujan does not define what he means by order. In his papers and notebooks, Ramanujan uses this word to mean "relative size" or "approximation" (for possibly large values of a parameter) or to describe how a certain function grows, with increasing $n$, say. See, for example [1], [2, pp. 78-128]. 
As $\epsilon$ increases, the value of $v_{m}$ in (2.4) decreases and the value of $v_{m}$ in (2.5) increases. Thus, the smallest possible value of $v_{m}$ is obtained when

$$
\epsilon=\frac{1}{2}-\frac{m-\sqrt{m^{2}-1}}{2 \sqrt{3}} .
$$

But, if we recall the definition of $\epsilon$, the value of $\epsilon$ is chosen so that $0<\epsilon<1$ and the two values in (2.3) are rational with denominator $m$. In other words, we do not have the liberty of choosing $\epsilon$ since it is completely determined by the value of $m$. So, one can regard "the order of $v_{m}$ is minimum" to mean that for the values of $m$ for which (2.17) holds (or equivalently, the right side of (2.16) is a rational number with denominator $m), v_{m}$ takes the smallest possible value, which is (2.15). Here, we remark that by (2.12) and (2.13), (2.16) should be written as

$$
\epsilon_{m}=\frac{1}{m}\left(\frac{m-1}{2}-\sqrt{\frac{m^{2}-1}{12}}\right) \quad \text { or } \quad \frac{1}{m}\left(\frac{m+1}{2}-\sqrt{\frac{m^{2}-1}{12}}\right) .
$$

Below (2.16) on the same page, Ramanujan also discusses the maximum order of $v_{m}$. the maximum order of $v_{m}$ can be got by making $\epsilon$ as small as possible in (2.4), that is by making $m \epsilon_{m}$ as nearly equal to $m(3-\sqrt{3}) / 6$ as possible from below. Hence

$$
\epsilon_{m}=\frac{1}{m}\left(\frac{m}{2}-\sqrt{\frac{m^{2}+2}{12}}\right)
$$

and

$$
v_{m}=\frac{\left(m^{2}-1\right)}{6 m^{3}} \sqrt{\frac{m^{2}+2}{3}} .
$$

We first note that

$$
m \frac{3-\sqrt{3}}{6}=\frac{m}{2}-\sqrt{\frac{m^{2}}{12}} \text { and } \frac{m}{2}-\sqrt{\frac{m^{2}+1}{12}}
$$

cannot be integers. Now, we consider those values of $m$ for which

$$
\frac{m}{2}-\sqrt{\frac{m^{2}+2}{12}}
$$

are integers (it is easy to see that $m$ is odd, and $m=5,19, \ldots$ ). Then, it can be easily verified that

$$
\left[\frac{m}{2}-\sqrt{\frac{m^{2}}{12}}\right]=\left[\frac{m}{2}-\sqrt{\frac{m^{2}+1}{12}}\right]=\left[\frac{m}{2}-\sqrt{\frac{m^{2}+2}{12}}\right]=\frac{m}{2}-\sqrt{\frac{m^{2}+2}{12}} .
$$

So, $(2.21)$ is the closest integer to $m(3-\sqrt{3}) / 6$ from below, and the value of $\epsilon$, which is

$$
\sqrt{\frac{m^{2}+2}{12}}-\sqrt{\frac{m^{2}}{12}}
$$

seems to be as small as possible. Since $\epsilon$ is small enough to apply (2.8), the value in (2.21) is $m \epsilon_{m}$, which verifies (2.19). 
We naturally ask whether or not we can approximate $m \epsilon_{m}$ from above in a similar fashion, by making $\epsilon$ very close to 1 . First, it can be easily verified that, for $0 \leq i \leq 3$,

$$
\frac{m}{2}-\sqrt{\frac{m^{2}-i}{12}}
$$

does not take any integer values. If we consider those values of $m$ for which

$$
\frac{m}{2}-\sqrt{\frac{m^{2}-4}{12}}
$$

are integers, then $m$ is even and

$$
\left[\frac{m}{2}-\sqrt{\frac{m^{2}}{12}}+1\right]=\frac{m}{2}-\sqrt{\frac{m^{2}-4}{12}} .
$$

So, the value of $1-\epsilon$ is

$$
\sqrt{\frac{m^{2}}{12}}-\sqrt{\frac{m^{2}-4}{12}}
$$

Also, we have

$$
\epsilon_{m}=\frac{m}{2}-\sqrt{\frac{m^{2}-4}{12}} \text { and } v_{m}=\frac{m^{2}+2}{6 m^{3}} \sqrt{\frac{m^{2}-4}{3}} .
$$

As mentioned earlier, Ramanujan did not define the maximum order of $v_{m}$. Thus, it is not obvious why he only considered $\epsilon$ as small as possible. However, we emphasize that the value in (2.19) is very useful when we examine the periodicity of $v_{m}$ in the next section. This concludes the first section of Ramanujan's partial manuscript.

\section{The Periodicity of $v_{m}$}

We quote Ramanujan from the first portion of his second and last section.

From the table we find that

$$
v_{5}=v_{10}=v_{15}=v_{20}=v_{25}=v_{30}=v_{35}=v_{40} .
$$

which suggests the question: How to determine the maximum value of $k$ in order that

$$
v_{m}=v_{2 m}=v_{3 m}=\cdots=v_{k m} .
$$

From (2.16) and (2.19) we see that

$$
k \ngtr\left[\frac{x}{m}\right]
$$

where

$$
\frac{1}{x}\left(\frac{x+1}{12}-\sqrt{\frac{x^{2}-1}{12}}\right)=\frac{1}{m}\left(\frac{m}{2}-\sqrt{\frac{m^{2}+2}{12}}\right) .
$$

That is

$$
k \ngtr\left[\sqrt{3 m^{2}+6}-1\right] .
$$

It can also be shown that, if $3 m^{2}+6$ is a perfect square, then

$$
k=\sqrt{3 m^{2}+6}-1 \text {. }
$$


The actual value of $k$ can be got from (2.11) and (2.16). Thus

$$
k=\left[\frac{x}{m}\right] \text {, }
$$

where

$$
\frac{1}{x}\left(\frac{x+1}{12}-\sqrt{\frac{x^{2}-1}{12}}\right)=\frac{1}{m}\left[\frac{m+1}{12}-\sqrt{\frac{m^{2}-1}{12}}\right] .
$$

We note that 5 from (3.1) is the smallest value of $m$ for which the value in (2.19) is a rational with denominator $m$. The table to which Ramanujan refers is a table of the first 50 values of $v_{m}$, which is given at the end of his partial manuscript and which is also given at the end of the present paper. The periodicity of values given in (3.1) is remarkable. Observe that, by (2.4) and (2.5), if values of $v_{m}$ are identical for certain values of $m$, then the corresponding values of $\epsilon_{m}$ must be identical.

From (2.11), recall that for any $m$

$$
\epsilon_{m}=\frac{1}{m}\left[\frac{m+1}{2}-\sqrt{\frac{m^{2}-1}{12}}\right]
$$

or using the least integer function

$$
\epsilon_{m}=\frac{1}{m}\left\lceil\frac{m-1}{2}-\sqrt{\frac{m^{2}-1}{12}}\right\rceil .
$$

We first examine the following two functions. For $x>1$,

$$
f_{1}(x):=\frac{1}{x}\left(\frac{x+1}{2}-\sqrt{\frac{x^{2}-1}{12}}\right) \quad \text { and } \quad f_{2}(x):=\frac{1}{x}\left(\frac{x-1}{2}-\sqrt{\frac{x^{2}-1}{12}}\right) .
$$

An elementary calculation shows that

$$
f_{1}^{\prime}(x)=-\frac{1}{2 x^{2}}-\frac{1}{12 x^{2}}\left(\frac{x^{2}-1}{12}\right)^{-1 / 2}<0, \quad f_{2}^{\prime}(x)=\frac{1}{2 x^{2}}-\frac{1}{12 x^{2}}\left(\frac{x^{2}-1}{12}\right)^{-1 / 2}>0 .
$$

Thus, $f_{1}(x)$ is monotonically decreasing and $f_{2}(x)$ is monotonically increasing. Also, we see that

$$
f_{2}(x)=\frac{1}{2}-\frac{1}{2 x}-\sqrt{\frac{1}{12}-\frac{1}{12 x^{2}}}<\frac{3-\sqrt{3}}{6}<f_{1}(x)=\frac{1}{2}+\frac{1}{2 x}-\sqrt{\frac{1}{12}-\frac{1}{12 x^{2}}} .
$$

Now, we first verify (3.3) and (3.5). We can see that Ramanujan only considered those values of $m$ for which

$$
\epsilon_{m}=\frac{1}{m}\left(\frac{m}{2}-\sqrt{\frac{m^{2}+2}{12}}\right),
$$

as in (2.19). Suppose that we have the sequence

$$
v_{m}=v_{2 m}=v_{3 m}=\cdots=v_{k m},
$$

which implies that

$$
\epsilon_{m}=\epsilon_{2 m}=\epsilon_{3 m}=\cdots=\epsilon_{k m} .
$$


In fact, Ramanujan's equation (3.4) is incorrect. The right-hand side of (3.4) is

$$
\frac{1}{m}\left(\frac{m}{2}-\sqrt{\frac{m^{2}+2}{12}}\right)=\frac{1}{2}-\sqrt{\frac{1}{12}+\frac{1}{6 m^{2}}}<\frac{3-\sqrt{3}}{6},
$$

while the left-hand side of (3.4), by (3.9), is equal to

$$
\frac{1}{2}+\frac{1}{2 x}-\sqrt{\frac{1}{12}-\frac{1}{12 x^{2}}}=f_{1}(x)>\frac{3-\sqrt{3}}{6} .
$$

Clearly, (3.11) and (3.12) are incompatible. This caused confusion for the writer of the first note appended to Ramanujan's article. She writes, "I don't see where eqn (3.5) comes from, eg $m=5, k=8$ (quoted on p. 3) does not come from the value of $k$ given $\left[\frac{x}{m}\right]$, as $x$ is negative."

We see that we must have

$$
\frac{1}{x}\left(\frac{x-1}{2}-\sqrt{\frac{x^{2}-1}{12}}\right)=\frac{1}{m}\left(\frac{m}{2}-\sqrt{\frac{m^{2}+2}{12}}\right)=\epsilon_{m}
$$

instead. Since $\epsilon_{m}=\epsilon_{k m}$, by (2.11) and (3.13),

$\frac{1}{x}\left(\frac{x-1}{2}-\sqrt{\frac{x^{2}-1}{12}}\right)=\frac{1}{k m}\left[\frac{k m+1}{2}-\sqrt{\frac{k^{2} m^{2}-1}{12}}\right] \geq \frac{1}{k m}\left(\frac{k m-1}{2}-\sqrt{\frac{k^{2} m^{2}-1}{12}}\right)$.

Since $f_{2}(x)$ is monotonically increasing, it follows that $x \geq k m$, which proves (3.3).

Now, we solve (3.13). Let

$$
\alpha=\frac{1}{m}\left(\frac{m}{2}-\sqrt{\frac{m^{2}+2}{12}}\right) .
$$

Then,

$$
\begin{aligned}
& \frac{x-1}{2}-\sqrt{\frac{x^{2}-1}{12}}=\alpha x, \\
& (1-2 \alpha) x-1=2 \sqrt{\frac{x^{2}-1}{12},} \\
& (1-2 \alpha)^{2} x^{2}-2(1-2 \alpha) x+1=\frac{x^{2}-1}{3}, \\
& \left(3(1-2 \alpha)^{2}-1\right) x^{2}-6(1-2 \alpha) x+4=0 .
\end{aligned}
$$

Thus,

$$
\begin{aligned}
x & =\frac{6(1-2 \alpha) \pm \sqrt{36(1-2 \alpha)^{2}-16\left(3(1-2 \alpha)^{2}-1\right)}}{6(1-2 \alpha)^{2}-2} \\
& =\frac{3(1-2 \alpha) \pm \sqrt{1+12 \alpha-12 \alpha^{2}}}{3(1-2 \alpha)^{2}-1} .
\end{aligned}
$$


Since $\alpha=\frac{1}{2}-\frac{1}{m} \sqrt{\frac{m^{2}+2}{12}}$, we easily find that

$$
\begin{aligned}
& 1+12 \alpha-12 \alpha^{2}=4-12\left(\frac{1}{2}-\alpha\right)^{2}=\frac{3 m^{2}-2}{m^{2}} \\
& 3(1-2 \alpha)=\frac{\sqrt{3 m^{2}+6}}{m}, \\
& 3(1-2 \alpha)^{2}-1=2-12 \alpha+12 \alpha^{2}=12\left(\alpha-\frac{1}{2}\right)^{2}-1=\frac{2}{m^{2}} .
\end{aligned}
$$

Hence, we deduce that

$$
\frac{x}{m}=\frac{\sqrt{3 m^{2}+6}+\sqrt{3 m^{2}-2}}{2} .
$$

However, by $(3.10)$ we see that $\left(m^{2}+2\right) / 3$ is a perfect square, which is equivalent to $3 m^{2}+6$ being a perfect square. Thus,

$$
k \leq\left[\frac{x}{m}\right]=\sqrt{3 m^{2}+6}-1,
$$

which verifies (3.5) (in fact, we do not need the greatest integer function in (3.5)).

In (3.6) and (3.7), Ramanujan concludes that, in fact, $\left[\frac{x}{m}\right]$ is the exact value of $k$ for all $m$. We now give a proof for the general case (3.7), which naturally implies (3.6). First, we see that (3.8) should be replaced by the pair of relations, by (3.9),

$$
\frac{1}{x}\left(\frac{x+1}{12}-\sqrt{\frac{x^{2}-1}{12}}\right)=\frac{1}{m}\left[\frac{m+1}{12}-\sqrt{\frac{m^{2}-1}{12}}\right]>\frac{3-\sqrt{3}}{6}
$$

or

$$
\frac{1}{x}\left(\frac{x-1}{12}-\sqrt{\frac{x^{2}-1}{12}}\right)=\frac{1}{m}\left[\frac{m+1}{12}-\sqrt{\frac{m^{2}-1}{12}}\right]<\frac{3-\sqrt{3}}{6} .
$$

In order to prove (3.7), we first need to show that $k \leq\left[\frac{x}{m}\right]$ for arbitrary $m$. In the case of (3.15), we can use the same argument from the proof of (3.5). For the case of (3.14), if we assume $\epsilon_{m}=\epsilon_{2 m}=\cdots=\epsilon_{k m}$, then we have

$$
\frac{1}{x}\left(\frac{x+1}{12}-\sqrt{\frac{x^{2}-1}{12}}\right)=\frac{1}{k m}\left[\frac{k m+1}{12}-\sqrt{\frac{k^{2} m^{2}-1}{12}}\right] \leq \frac{1}{k m}\left(\frac{k m+1}{12}-\sqrt{\frac{k^{2} m^{2}-1}{12}}\right) .
$$

Since $f_{1}(x)$ is monotonically decreasing, we conclude that $k m \leq x$, or $k \leq\left[\frac{x}{m}\right]$.

We now show that for any $1 \leq t \leq\left[\frac{x}{m}\right], \epsilon_{m}=\epsilon_{t m}$, which proves (3.7). We first consider those values of $m$ for which (3.14) holds. Since all the rational numbers with denominator $t m$ include the rational numbers with denominator $m$, we have $v_{t m} \geq v_{m}$. Since $\epsilon_{m}>(3-\sqrt{3}) / 6$ and the function $f(x)=x(1-x)(1-2 x)$ is decreasing on the interval $[(3-\sqrt{3}) / 6,1]$, we have $\epsilon_{t m} \leq \epsilon_{m}$. On the other hand, since $f_{1}(x)$ is decreasing, by $(3.14)$,

$$
\epsilon_{m}=\frac{t}{t m}\left[\frac{m+1}{12}-\sqrt{\frac{m^{2}-1}{12}}\right] \leq \frac{1}{t m}\left(\frac{t m+1}{12}-\sqrt{\frac{t^{2} m^{2}-1}{12}}\right) .
$$


Thus, we have

$$
t\left[\frac{m+1}{12}-\sqrt{\frac{m^{2}-1}{12}}\right] \leq\left[\frac{t m+1}{12}-\sqrt{\frac{t^{2} m^{2}-1}{12}}\right]
$$

which implies that $\epsilon_{m} \leq \epsilon_{t m}$, upon dividing both sides above by $t m$. Hence, we have proved that $\epsilon_{m}=\epsilon_{t m}$ for all $1 \leq t \leq\left[\frac{x}{m}\right]$.

For those values of $m$ that satisfy (3.15), we apply a similar argument. Since $v_{t m} \geq v_{m}$ and the function $f(x)$ is increasing on the interval $[0,(3-\sqrt{3}) / 6]$, we have $\epsilon_{m} \leq \epsilon_{t m}$. Since $f_{2}(x)$ is increasing, by (3.15),

$$
\epsilon_{m}=\frac{t}{t m}\left[\frac{m+1}{12}-\sqrt{\frac{m^{2}-1}{12}}\right] \geq \frac{1}{t m}\left(\frac{t m-1}{12}-\sqrt{\frac{t^{2} m^{2}-1}{12}}\right) .
$$

Thus,

$$
t\left[\frac{m+1}{12}-\sqrt{\frac{m^{2}-1}{12}}\right] \geq\left\lceil\frac{t m-1}{12}-\sqrt{\frac{t^{2} m^{2}-1}{12}}\right]=\left[\frac{t m+1}{12}-\sqrt{\frac{t^{2} m^{2}-1}{12}}\right],
$$

which implies that $\epsilon_{t m} \leq \epsilon_{m}$, upon dividing both sides above by $t m$. Thus, we have shown that $\epsilon_{m}=\epsilon_{t m}$, which completes the proof of (3.7).

In summary, if $3 m^{2}+6$ is a perfect square, then we use (3.6) to calculate the length $k$ of the period. If $3 m^{2}+6$ is not a perfect square, then we use (3.7), with $x$ defined by (3.14) or (3.15), to calculate the period length $k$.

If $m=1$, then, by (3.6), $k=2$. In our initial calculations above, we had observed that $v_{1}=v_{2}=0$, but $v_{3} \neq 0$, and so Ramanujan's periodic assertion is corroborated in this case. Ramanujan then gives seven periodic sequences corresponding to the values $m=5,9,14,19,71,265,989$, with periods $8,5,12,32,122,458,1712$, respectively, namely,

$$
\begin{aligned}
& v_{5}=v_{10}=v_{15}=\cdots=v_{40}, \\
& v_{9}=v_{18}=v_{27}=\cdots=v_{45}, \\
& v_{14}=v_{28}=v_{42}=\cdots=v_{168}, \\
& v_{19}=v_{38}=v_{57}=\cdots=v_{608}, \\
& v_{71}=v_{142}=v_{213}=\cdots=v_{8662}, \\
& v_{265}=v_{530}=v_{795}=\cdots=v_{121370}, \\
& v_{989}=v_{1978}=v_{2967}=\cdots=v_{1693168} .
\end{aligned}
$$

In the penultimate line the last subscript is misprinted as 121670 in the lost notebook. The first, fourth, fifth, sixth, and seventh sequences arise from (3.6), but for the second and third, we must use (3.7) and (3.14) to determine the values $k=5$ and $k=12$, respectively.

It is interesting to examine how often $3 m^{2}+6$ is a perfect square. If we let $3 m^{2}+6=$ $n^{2}$ or $n^{2}-3 m^{2}=6$, then $n+m \sqrt{3}$ is an element of $\mathbb{Z}[\sqrt{3}]$ with norm 6 . Since $3+\sqrt{3}$ is such an element with positive smallest values of $n$ and $m$, and $2+\sqrt{3}$ is the fundamental unit of $\mathbb{Z}[\sqrt{3}]$, all the values of $n$ and $m$ generated by $(3+\sqrt{3})(2+\sqrt{3})^{r}$ with $r \in \mathbb{Z}$ are solutions. In fact, we can also show that they are the only solutions, using the LLM 
algorithm as described by J.P. Robertson [4, pp. 4, 5, 16]. We remark that the values $m=5,19,71,265,989$ are generated by $(3+\sqrt{3})(2+\sqrt{3})^{r}$ with $1 \leq r \leq 5$.

On the last page of his manuscript, Ramanujan gives the numerical value

$$
v_{\infty}=\frac{1}{6 \sqrt{3}}=.0962250 .
$$

He then provides a table of the first 50 values of $v_{m}$. All the given values are correct, except that the last recorded (sixth) digit of $v_{23}=v_{46}$ is 7 , not 8 as claimed by Ramanujan. More precisely, $v_{23}=v_{46}=.09616174899$.

We conclude with a couple of natural questions arising from Ramanujan's calculations.

(1) Ramanujan found that some periodic sequences have "large" values of $k$. Does there exist a sequence $\left\{v_{m}\right\}$ such that $k=k_{m} \rightarrow \infty$ ?

(2) Given an arbitrary positive integer $k$, does there exist a value of $v_{m}$ with periodic length $k$ ?

\begin{tabular}{|l|l|}
\hline$v_{1}=0$ & $v_{26}=.0955849$ \\
\hline$v_{2}=0$ & $v_{27}=.0960219$ \\
\hline$v_{3}=.0740741$ & $v_{28}=.0962099$ \\
\hline$v_{4}=.0937500$ & $v_{29}=.0961909$ \\
\hline$v_{5}=.0960000$ & $v_{30}=.0960000$ \\
\hline$v_{6}=.0925926$ & $v_{31}=.0958679$ \\
\hline$v_{7}=.0874436$ & $v_{32}=.0961304$ \\
\hline$v_{8}=.0937500$ & $v_{33}=.0962239$ \\
\hline$v_{9}=.0960219$ & $v_{34}=.0961734$ \\
\hline$v_{10}=.0960000$ & $v_{35}=.0960000$ \\
\hline$v_{11}=.0946657$ & $v_{36}=.0960219$ \\
\hline$v_{12}=.0937500$ & $v_{37}=.0961838$ \\
\hline$v_{13}=.0955849$ & $v_{38}=.0962239$ \\
\hline$v_{14}=.0962099$ & $v_{39}=.0961581$ \\
\hline$v_{15}=.0960000$ & $v_{40}=.0960000$ \\
\hline$v_{16}=.0952148$ & $v_{41}=.0961100$ \\
\hline$v_{17}=.0952575$ & $v_{42}=.0962099$ \\
\hline$v_{18}=.0960219$ & $v_{43}=.0962179$ \\
\hline$v_{19}=.0962239$ & $v_{44}=.0961448$ \\
\hline$v_{20}=.0960000$ & $v_{45}=.0960219$ \\
\hline$v_{21}=.0954541$ & $v_{46}=.0961617$ \\
\hline$v_{22}=.0957926$ & $v_{47}=.0962215$ \\
\hline$v_{23}=.0961617$ & $v_{48}=.0962095$ \\
\hline$v_{24}=.0962095$ & $v_{49}=.0961334$ \\
\hline$v_{25}=.0960000$ & $v_{50}=.0960960$ \\
\hline
\end{tabular}

TABLE 2. Table of Values for $v_{m}, 1 \leq m \leq 50$ 


\section{REFERENCES}

[1] S. Ramanujan, Highly composite numbers, Proc. London Math. Soc. (2) 14 (1915), 347-409.

[2] S. Ramanujan, Collected Papers, Cambridge University Press, Cambridge, 1927; reprinted by Chelsea, New York, 1962; reprinted by the American Mathematical Society, Providence, RI, 2000.

[3] S. Ramanujan, The Lost Notebook and Other Unpublished Papers, Narosa, New Delhi, 1988.

[4] J.P. Robertson, Solving the generalized Pell equation $x^{2}-D y^{2}=N$, www.jpr2718.org/pell.pdf

Department of Mathematics, University of Illinois, 1409 West Green Street, UrBANA, IL 61801, USA

E-mail address: berndt@illinois.edu

E-mail address: sunkim2@illinois.edu 